\section{Le réveil des cardiomyocytes adultes résidents}

\section{Une nouvelle piste de régénération cardiaque?}

\author{
Céline Mias ${ }^{1}$, Gaël Genet ${ }^{1}$, Atul Pathak ${ }^{1,2}$, \\ Jean-Michel Sénard ${ }^{1,2}$, Céline Galés ${ }^{1}$
}

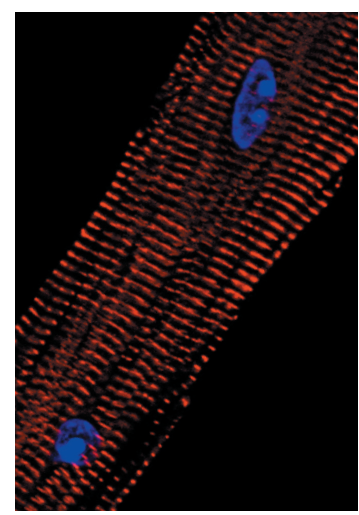
${ }^{1}$ Institut des maladies métaboliques et cardiovasculaires (I2MC), Inserm UMR 1048, université Toulouse III Paul Sabatier, Bâtiment L3,
1 , avenue Jean Poulhès, BP 84225, 31432 Toulouse Cedex 4, France ; ${ }^{2}$ Service de pharmacologie clinique, fédération des services de cardiologie, centre hospitalier universitaire de Toulouse, Toulouse, France.
celine.mias@inserm.fr

dérés comme bloqués dans un statut postmitotique et donc incapables de proliférer. Aussi, jusqu'à présent, les efforts en médecine cardiaque régénératrice ont porté essentiellement sur la thérapie cellulaire et l'utilisation des cellules souches, qu'il s'agisse de progéniteurs cardiaques, de cellules souches issues d'autres tissus que le cœur (cellules souches de la moelle osseuse, cellules satellites du muscle squelettique, etc.) ou de dérivés de cellules souches embryonnaires [1]. Cette stratégie n'a malheureusement abouti qu'à de modestes améliorations, voire aucune amélioration, de la fonction cardiaque. Ces approches de thérapie cellulaire restent limitées du fait des risques, encore mal évalués, inhérents aux cellules souches (différenciation inadaptée des cellules souches dans le tissu cible, potentiel tumoral, entre autres) [1]. Développer de nouvelles voies thérapeutiques s'avère donc essentiel en médecine régénératrice cardiaque. Dans ce contexte, des études récentes ont démontré que les CM adultes résidents sont capables d'entrer à nouveau dans le cycle cellulaire en conditions physiologiques, mais également sous l'influence de différents facteurs ou de manipulations géniques, et de participer à la réparation du tissu cardiaque endommagé. Pour que l'utilisation des CM résidents puisse émerger comme une approche thérapeutique fiable et d'envergure, il est indispensable de mieux comprendre ce processus de « reprogrammation » proliférative.

\section{La régénération cardiaque : un processus naturel d'exception chez certains poissons et amphibiens}

Bien que pratiquement inexistante chez les mammifères adultes, la capacité de régénération du tissu cardiaque subsiste cependant chez 
quelques rares vertébrés, mais s'avère très variable d'une espèce à l'autre. Elle est ainsi très élevée chez certains poissons (poisson zèbre) et modérée chez les amphibiens (axolotl). Le poisson zèbre constitue un excellent modèle d'étude de la régénération cardiaque à l'âge adulte, et permet des manipulations génétiques faciles [2]. Jusqu'à récemment, les mécanismes de régénération cardiaque induits par une résection partielle du myocarde ventriculaire chez le poisson zèbre faisaient intervenir des cellules progénitrices cardiaques, recrutées au sein de la lésion et qui se différenciaient en CM pour régénérer la structure tissulaire éliminée [3]. La contribution de cellules cardiaques non myocytaires d'origine épicardique a été suggérée par différents travaux, sans toutefois qu'un mécanisme de transdifférenciation de ces cellules en CM au niveau de la résection ne soit jamais clairement établi [2, 4]. Cependant, et contre toute attente, les travaux récents de Jopling et al. [5], utilisant une stratégie de traçage génétique spécifique de la lignée cardiomyocytaire, démontrent clairement que, chez le poisson zèbre, la grande majorité des cellules contractiles cardiaques formées durant le processus de régénération proviennent de CM préexistants. Ainsi, les progéniteurs cardiaques résidents ne participeraient que très modestement au processus de régénération cardiaque. Un mécanisme initial de dédifférenciation cellulaire serait impliqué dans la régénération cardiaque via les CM résidents. Ainsi, une perte de cohésion intercellulaire et une désorganisation importante de l'appareil contractile (sarcomères) seraient nécessaires à la réactivation du cycle cellulaire. D'un point de vue moléculaire, chez le poisson zèbre comme chez la salamandre, les CM en cours de régénération répriment l'expression des gènes sarcomériques, et induisent celle des gènes codant pour des protéines intervenant dans la progression du cycle cellulaire et, plus particulièrement, polo-like kinase 1 et mps 1 impliqués dans la mitose $[5,6]$. Si ces travaux démontrent clairement l'origine des CM dans la zone de régénération, il n'en reste pas moins vrai que les cellules cardiaques avoisinantes, d'origine épicardique, participent activement au processus de régénération, notamment en favorisant la néovascularisation, mais également en tant que source de facteurs mitogènes [3].

\section{Absence de régénération cardiaque naturelle chez les mammifères : un mythe ou un dogme qui tombe?}

\section{Antagonisme entre prolifération et cicatrisation : les modèles du poisson zèbre et de la souris}

Selon un dogme ancestral, le cœur de mammifères est incapable de se régénérer. Typiquement, et contrairement au poisson zèbre, les lésions cardiaques chez les mammifères adultes conduisent à la formation d'un zone cicatricielle constituée majoritairement de tissu fibreux qui perturbe la contraction cardiaque [4]. Chez les mammifères, l'absence de prolifération des CM est directement liée à la mise en place d'une cicatrice, elle-même déclenchée par la réponse inflammatoire aiguë. Cette réponse inflammatoire très précoce, outre qu'elle recrute des cellules immunitaires, active des fibroblastes. Ceux-ci se transforment en myofibroblastes qui sécrètent des quantités massives de collagène matriciel extracellulaire, assurant le comblement de la zone lésée et la formation de la cicatrice. Néanmoins, si la cicatrisation à elle seule empêche la prolifération des CM, on n'explique pas pourquoi, dans des modèles de poissons zèbres génétiquement modifiés et développant une cicatrice, la capacité régénératrice après résection cardiaque n'est pas abolie [7]. Or, plusieurs études récentes démontrent que les CM issus d'un cœur adulte de mammifères sont capables de se renouveler dans les conditions classiques d'homéostasie, mais également après atteinte tissulaire (Tableau I).

L'idée d'une régénération myocardique à partir d'un pool de cellules souches cardiaques capables de se différencier en cellules cardiaques contractiles n'a cessé de progresser ces dernières années. En particulier, une étude génétique élégante, menée chez la souris et utilisant un système de marquage fluorescent inductible des CM préexistants, a permis de montrer l'existence de capacités régénératives cardiaques endogènes, notamment en réponse à un stress ischémique ou barométrique. Mais ce renouvellement des CM s'expliquerait davantage par leur différenciation à partir de cellules progénitrices cardiomyogéniques que par la prolifération de CM préexistants [8]. Ce mécanisme reste cependant extrêmement limité puisqu'incapable de suppléer au dysfonctionnement du tissu cardiaque ou d'empêcher le développement d'une hypertrophie/ fibrose compensatoire. Cela explique les efforts considérables de thérapie cellulaire entrepris aujourd'hui pour renforcer le pool de cellules souches au niveau de la lésion cardiaque par un apport cellulaire exogène de cellules souches ou progéniteurs cardiaques.

Si le dogme selon lequel les capacités de régénération cardiaque sont négligeables chez les mammifères adultes est bien réel, il n'en est pas de même au cours du développement. En effet, chez la souris, le cœur embryonnaire et néonatal possède une remarquable capacité de régénération $[9,10]$. Le laboratoire du Pr. Olson a ainsi démontré que la résection d'une partie de l'apex chez une souris un jour après sa naissance s'accompagne d'une régénération de la zone d'apectomie par prolifération des CM résidents, comme on l'observe chez le poisson zèbre. Cependant, dès le $7^{\mathrm{e}}$ jour de vie, une telle régénération n'est plus possible et un mécanisme classique de cicatrisation se met en place. La possibilité de régénération du cœur de mammifère, qui existe au stade néonatal, disparaît donc rapidement au cours de la maturation postnatale des CM, et est parfaitement corrélé avec la disparition progressive de l'index prolifératif du CM au cours de la maturation.

\section{Un renouvellement des CM chez l'homme?}

Qu'en est-il chez l'homme? Selon de nombreux travaux, les CM humains seraient capables de synthèse d'ADN en 


\begin{tabular}{|c|c|c|c|c|}
\hline Espèce & Modèles & Mécanismes & Effets biologiques observés & Réf. \\
\hline Poisson zèbre & $\begin{array}{l}\text { Apectomie } \\
\text { Traçage génétique du lignage } \\
\text { cardiomyocytaire }\end{array}$ & $\begin{array}{l}\uparrow \text { Gènes du cycle } \\
\text { cellulaire } \\
\text { polo-like kinase } 1 \\
(p l k 1) \text {; mps } 1\end{array}$ & $\begin{array}{l}\text { Régénération cardiaque } \\
\text { par dédifférenciation de CM résidents }\end{array}$ & [5] \\
\hline $\begin{array}{l}\text { Souris } \\
\text { néonatale }\end{array}$ & Apectomie & ND & $\begin{array}{l}\text { Corrélation index prolifératif des CM (néonatals } \\
\text { versus adultes)/régénération cardiaque }\end{array}$ & [10] \\
\hline Souris adulte & $\begin{array}{l}\text { Stimulation FGF-1 } \\
+ \text { inhibiteur p38 }\end{array}$ & ND & $\begin{array}{l}+++ \text { Division des CM adultes } \\
+++ \text { Angiogenèse } \\
+++ \text { Fonction cardiaque après IDM }\end{array}$ & [14] \\
\hline Souris adulte & Stimulation périostine & $\begin{array}{l}\text { Voie PI3K/Akt } \\
\text { dans la prolifération } \\
\text { des CM adultes }\end{array}$ & $\begin{array}{l}\text { +++ Réentrée des CM matures dans le cycle } \\
\text { cellulaire } \\
+++ \text { Réparation du tissu cardiaque } \\
+++ \text { Angiogenèse } \\
+++ \text { Fonction cardiaque après IDM }\end{array}$ & [15] \\
\hline Souris adulte & Stimulation neuréguline 1 & $\begin{array}{l}\text { Voie PI3K } \\
\text { dans la prolifération } \\
\text { des CM adultes }\end{array}$ & $\begin{array}{l}+++ \text { Division des CM mononucléés } \\
+++ \text { Réentrée des CM dans le cycle cellulaire } \\
+++ \text { Réparation du tissu cardiaque } \\
+++ \text { Fonction cardiaque après IDM }\end{array}$ & [13] \\
\hline $\begin{array}{l}\text { Homme } \\
\text { adulte }\end{array}$ & Incorporation ${ }^{14} \mathrm{C}$ & ND & $\begin{array}{l}\text { Renouvellement des CM résidents } \\
\text { ( } 45 \% \text { au cours d'une vie humaine) } \\
\downarrow \text { renouvellement des CM avec l'âge }\end{array}$ & [11] \\
\hline $\begin{array}{l}\text { Homme } \\
\text { adulte }\end{array}$ & $\begin{array}{l}\text { Examens postmortem de cœurs de } \\
\text { patients atteints de cancer et traités } \\
\text { à l'iododéoxyuridine }\end{array}$ & ND & $\begin{array}{l}\text { Prédiction du taux de synthèse d'ADN dans les CM } \\
\text { adultes : } 22 \% \text { / an }\end{array}$ & [12] \\
\hline
\end{tabular}

Tableau I. Résultats des études mettant en évidence le pouvoir prolifératif des CM résidents et leur potentiel de régénération cardiaque dans différentes espèces. IDM, infarctus du myocarde ; ND, non déterminé ; CM : cardiomyocytes.

réponse à un stress cardiaque. Néanmoins, elle se produit en l'absence totale de division cellulaire, augmentant la ploïdie des CM, et ne reflète donc pas l'état prolifératif de cette cellule. Plusieurs groupes ont essayé de mesurer indirectement l'index prolifératif des CM chez I'homme, mais ces techniques réalisées sur tissu, et donc peu fiables, rendent l'interprétation des résultats délicate. Récemment, Bergmann et al. [11] ont utilisé une méthode de datation des CM en tirant profit de l'incorporation de carbone $14\left({ }^{14} \mathrm{C}\right)$ dans I'ADN génomique de certaines populations exposées aux radiations lors de tests nucléaires pendant la guerre froide. Après extraction de l'ADN et analyse précise de la ploïdie des CM, les auteurs démontrent pour la première fois l'existence d'un renouvellement des CM résidents au cours de la vie adulte. Le taux de renouvellement diminue avec l'âge : il est de $1 \%$ par an à 25 ans et de seulement $0,45 \%$ par an à 75 ans. Le calcul prédit ainsi que $45 \%$ des CM seront renouvelés au cours d'une vie humaine. Dans une seconde étude menée par Kajstura et al. [12], le taux de synthèse d'ADN dans les $C M$ a été calculé en analysant postmortem des cœurs de patients atteints de cancer et ayant été traités à l'iododéoxyuridine. Cet analogue de la thymidine, qui s'incorpore à l'ADN au cours de la réplication, a pu être détecté par des méthodes d'immunohistologie dans les CM des cœurs de patients traités. L'analyse mathématique poussée des résultats indique ainsi un taux de renouvellement des CM incroyable, de $22 \%$ par an, approchant celui des cellules cardiaques non myocytaires (fibroblastes: $20 \%$ par an ; cellules endothéliales : $13 \%$ par an) dont on sait qu'elles se renouvellent régulièrement au cours de la vie, et même celui de certains sarcomes. Les processus de réparation de l'ADN mis en jeu lors des traitements radio-ionisants, ainsi que la polyploïdie des CM, ignorée dans cette étude, contribuent certainement à une surestimation du taux de renouvellement de ces cellules. Même s'il demeure difficile de chiffrer le taux de renouvellement exact des CM au cours de la vie humaine, ces deux études apportent toutefois la preuve de l'existence d'une plasticité des CM résidents permettant un renouvellement des cellules contractiles cardiaques.

\section{Peut-on programmer le renouvellement des CM résidents?}

Actuellement, les mécanismes moléculaires permettant le renouvellement des CM à partir des CM préexistants restent encore énigmatiques, mais l'activation des voies 


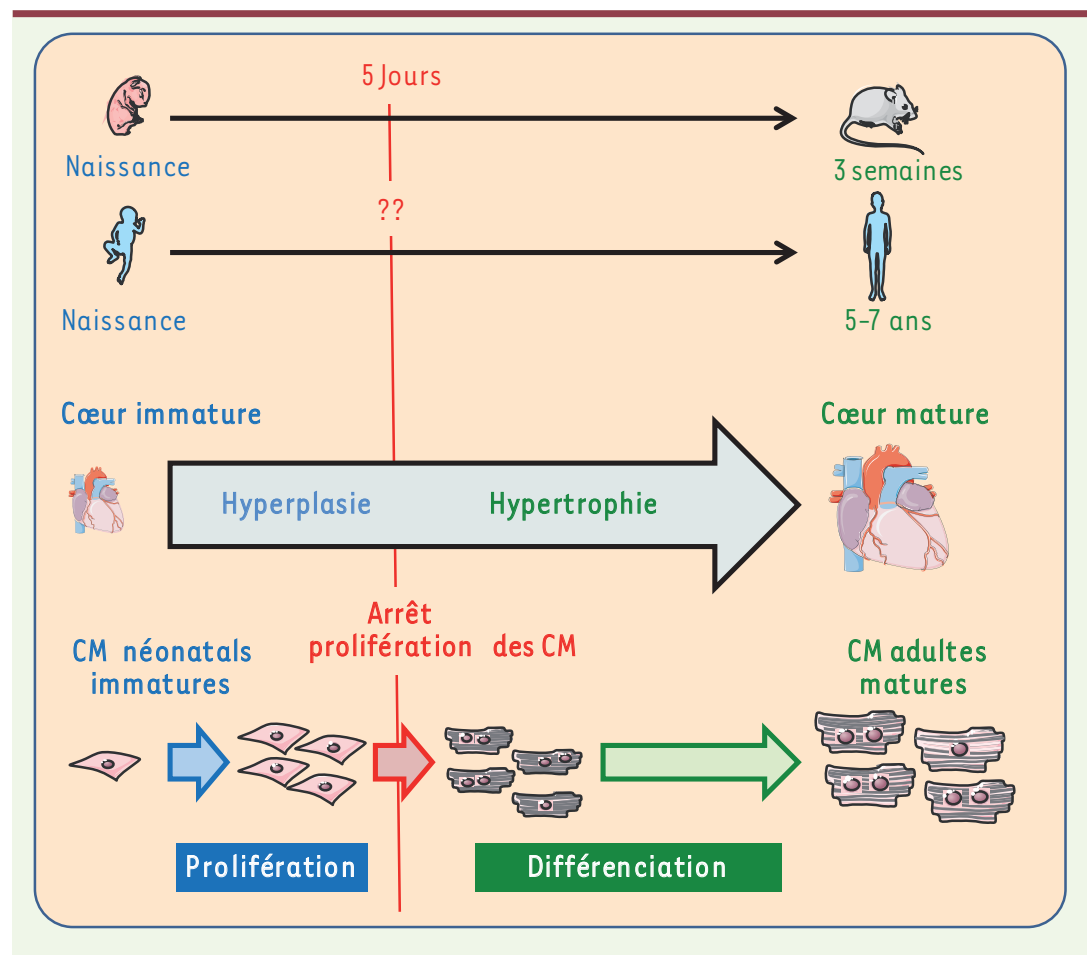

Figure 1. Développement cardiaque postnatal chez les mammifères. À la naissance, le cœur des mammifères n'est pas encore mature. La maturation cardiaque ne se produit qu'après la naissance et sa durée varie d'une espèce à l'autre, pouvant aller de 20 jours chez les rongeurs jusqu'à 5 à 7 ans chez l'homme. Cette maturation correspond à une augmentation de la masse cardiaque et fait intervenir deux mécanismes distincts : I'hyperplasie, correspondant à une prolifération accrue des cardiomyocytes (CM) au stade néonatal précoce, suivie rapidement d'une phase d'hypertrophie au cours de laquelle les CM cessent de proliférer (J5 postnatal chez les rongeurs) et augmentent uniquement leur taille, mais subissent également un processus de différenciation cellulaire pendant lequel ils acquièrent une morphologie adulte caractéristique de brique.

\section{Pourquoi les CM de mammifères perdent-ils leur statut prolifératif au stade adulte?}

mitogènes actives sur les CM représente une piste privilégiée. Trois puissants mitogènes, le facteur de croissance des fibroblastes (FGF-1), la périostine et la neuréguline l (NRGl), sont capables d'induire la réentrée des CM adultes dans le cycle cellulaire, permettant leur division/prolifération à la fois in vivo et in vitro [13-15], et ce malgré une nette diminution de l'index mitotique mesuré in vivo [13] (Tableau l). Bien que ces études démontrent un effet thérapeutique bénéfique de l'administration de ces molécules sur la fonction cardiaque après dommage ischémique, aucun lien causal n'a cependant été clairement établi entre la prolifération des CM résidents et l'effet protecteur cardiaque de ces molécules. Notamment, l'impact bénéfique de ces molécules sur le réseau vasculaire n'est pas à exclure. Les effets de NRGl sur le cycle cellulaire dépendent de l'expression de son récepteur ErbB4 dans les CM et mettent en jeu la voie classique de signalisation de la phosphoinositide 3-kinase (PI3K) [13], qui relaye aussi l'action de la périostine [15]. De façon intéressante, NRGl stimule exclusivement la division des populations de CM mononucléés et ne modifie pas la population binucléée. Ainsi, au sein des CM mononucléés, 50 \% sont capables de se diviser et de contribuer à l'accroissement de la population, alors que les autres $50 \%$ évoluent vers des cellules binucléées et un statut différencié postmitotique. Jusqu'à présent, l'ensemble des traitements visant à déclencher la prolifération des CM résidents ont été testés avec des CM sains, mais encore faut-il déterminer s'ils seront aussi efficaces sur des CM pathologiques. En effet, l'expression des récepteurs ErbB4 des CM diminue fortement dans différentes pathologies cardiaques [16] et pourrait expliquer la dichotomie entre les études d'induction de prolifération des CM in vitro par la NRGl et le faible taux de prolifération des CM résidents au sein d'un tissu cardiaque ischémique in vivo [13].

\section{Hyperplasie et hypertrophie : deux mécanismes d'augmentation de la masse cardiaque}

Au cours du développement cardiaque, deux mécanismes distincts contribuent à l'augmentation de la masse cardiaque : I'hyperplasie, qui correspond à une augmentation du nombre de CM via leur prolifération, caractérise les stades embryonnaire et néonatal précoce ; une phase d'hypertrophie postnatale la suit, au cours de laquelle les CM cessent de proliférer et seule leur taille augmente [17] (Figure 1). La prolifération des fibroblastes d'origine épicardique contribue également de façon significative à l'augmentation de la masse cardiaque pendant la phase embryonnaire [18]. Contrairement à la majorité des cellules de l'organisme, la maturation du CM ne se produit qu'après la naissance, et sa durée varie énormément d'une espèce à l'autre, pouvant aller de 20 jours chez les rongeurs [19] jusqu'à 5 à 7 ans chez l'homme [20]. D'un point de vue architectural, la maturation du CM consiste en la différenciation d'une cellule néonatale immature de type mésenchymateuse en une cellule mature adulte présentant une forme très caractéristique en brique. Chez les rongeurs, la maturation postnatale des CM comprend généralement une phase de prolifération très active durant les cinq premiers jours suivant la naissance; audelà, les CM perdent progressivement et totalement au bout de dix jours leur capacité proliférative [17].

La perte du pouvoir prolifératif des CM durant leur maturation est attribuée à leur sortie du cycle cellulaire 


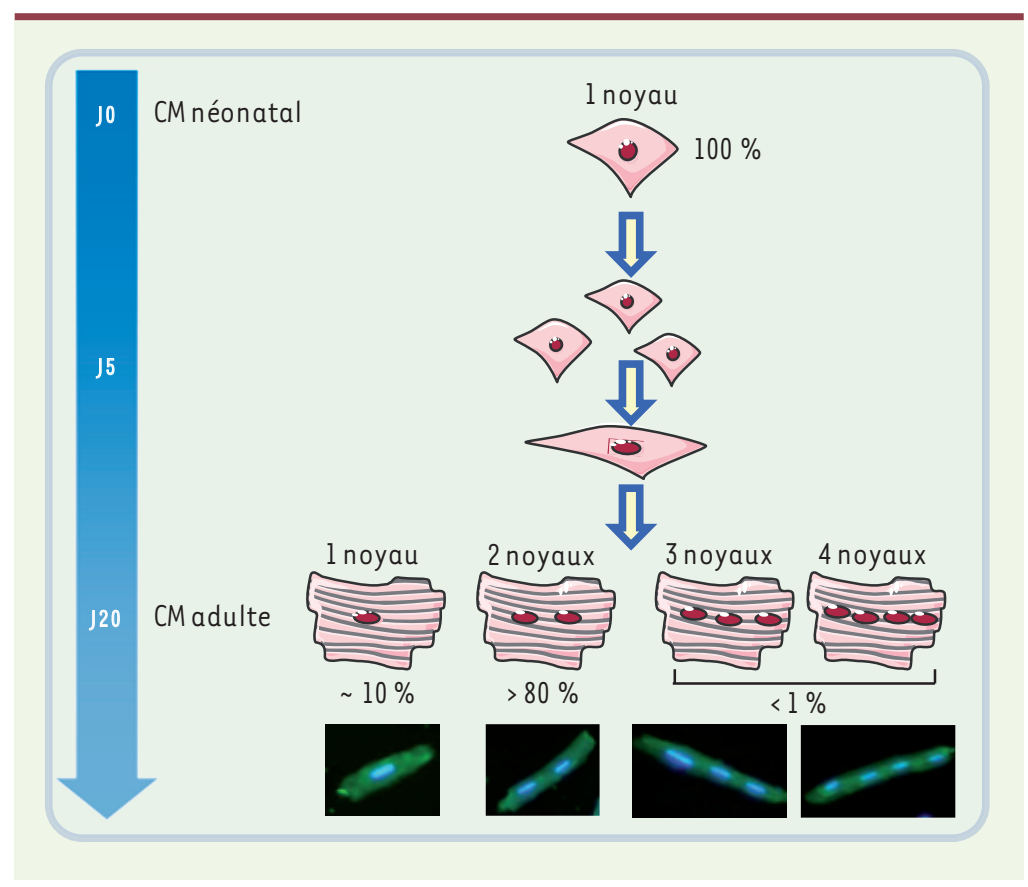

Figure 2. État de polynucléation des CM au cours de la maturation postnatale chez les rongeurs. À la naissance, le cœur des rongeurs présente une population unique de CM mononucléés, caractérisés par un fort pouvoir de division ; au cours de la maturation, ceux-ci donnent progressivement naissance à une population de CM binucléés postmitotiques majoritaires (> $80 \%)$, une population de CM mononucléés ( $10 \%)$, mais également à des CM tri- et tétranucléés très peu représentés ( $<1 \%)$.

[21] ; elle semble directement liée à la diminution des CM mononucléés. $\varepsilon n$ effet, à la naissance, le cœur des rongeurs contient une population unique de $\mathrm{CM}$ mononucléés, très prolifératifs. Ceux-ci maturent progressivement en une population majoritaire de CM binucléés postmitotiques (> $80 \%$ ), en CM mononucléés ( $10 \%)$ mais également en rares CM tri- et tétranucléés $(<1 \%)[17,22]$ (Figure 2). Une fois établies, les proportions de ces différentes populations de CM restent stables tout au long de la vie quelles que soient les situations physiologiques et/ou physiopathologiques [23]. Ces CM matures présentent un statut prolifératif bien particulier puisqu'ils sont bloqués dans les phases G0/ Gl (85\%) et G2/M (15\%) du cycle [24, 25] (Figure 3). Les CM binucléés correspondent à un stade de différenciation terminale et sont incapables de ré-entrer dans le cycle cellulaire. En accord avec ces résultats, NRGl permet la division des CM mononucléés mais n'a aucun effet sur les CM matures binucléés [13]. De la même façon, I'index mitotique de la population de CM mononucléés du cœur de salamandre est plus élevé que celui des CM binucléés [26]. Si la population de CM matures mononucléés est la seule capable de proliférer chez les rongeurs, qu'en est-il chez l'homme, espèce chez laquelle le rapport entre CM mononucléés (> $70 \%$ ) et binuclées $(25 \%)$ est inversé [22] ? Doiton en conclure que le cœur humain est doté d'un potentiel régénératif supérieur? Ceci pourrait-il expliquer pourquoi un plus grand nombre de travaux décrivant la réentrée des CM adultes dans le cycle cellulaire concernent le cœur humain? \&t qu'en est-il du potentiel mitotique des autres populations de CM tri- et tétranucléés, qui, bien que minori- taires, sont représentées chez l'homme et chez les rongeurs?

\section{Les acteurs moléculaires de la différenciation} cardiomyocytaire

D'un point de vue mécanistique, lors de la transition entre la phase d'hyperplasie et la phase d'hypertrophie, le CM réprime progressivement et significativement les activateurs du cycle cellulaire (cycline D/ CDK4-CDK6), alors qu'il induit de façon concomitante les inhibiteurs ( $\mathrm{Rb}$ [rétinoblastome], p21, p27) (Figure 3) [24, 25, 27]. La modulation importante de l'expression des acteurs de la phase Gl est prédominante, expliquant que la majorité des CM soient bloqués en phase $G l$ avant d'entrer dans le processus de différenciation. II n'est donc pas étonnant que de nombreux modèles murins transgéniques, réprimant l'expression des inhibiteurs du cycle cellulaire ou surexprimant ses activateurs, aient permis d'induire le cycle cellulaire des CM résidents et, de fait, de restimuler leur prolifération $[24,28,29]$. Toutefois, les conséquences de ces manipulations de la prolifération des CM en termes d'amélioration fonctionnelle cardiaque ont été peu convaincantes.

Outre les modifications du cycle cellulaire, la maturation des CM chez les mammifères s'accompagne de la mise en place d'une structure élaborée de l'appareil contractile à même de résister à la pression importante qu'entraîne à la naissance la mise en place de la circulation pulmonaire. Ce réseau de myofibrilles contractiles, important dans les CM adultes, pourrait également constituer un frein physique important au processus de division cellulaire qui nécessite une scission du réseau d'actine. Ce réseau est moins élaboré dans les CM néonataux des rongeurs ou adultes du poisson zèbre, ce qui pourrait expliquer en partie leur contribution plus importante à la régénération cardiaque. Dans le même esprit, certains groupes ont rapporté des anomalies de la phase finale de cytokinèse ${ }^{1}$ des $C M$, avec en particulier une délocalisation de l'aniline au niveau de l'anneau de scission du fuseau mitotique, nécessaire à la division des contenus nucléaire et cytoplasmique entre les deux cellules filles [30].

${ }^{1}$ La cytokinèse est l'étape finale de la division. Elle aboutit à l'individualisation des deux cellules filles. La séparation résulte de la contraction d'un anneau contenant de l'actine, de la myosine et d'autres protéines. 


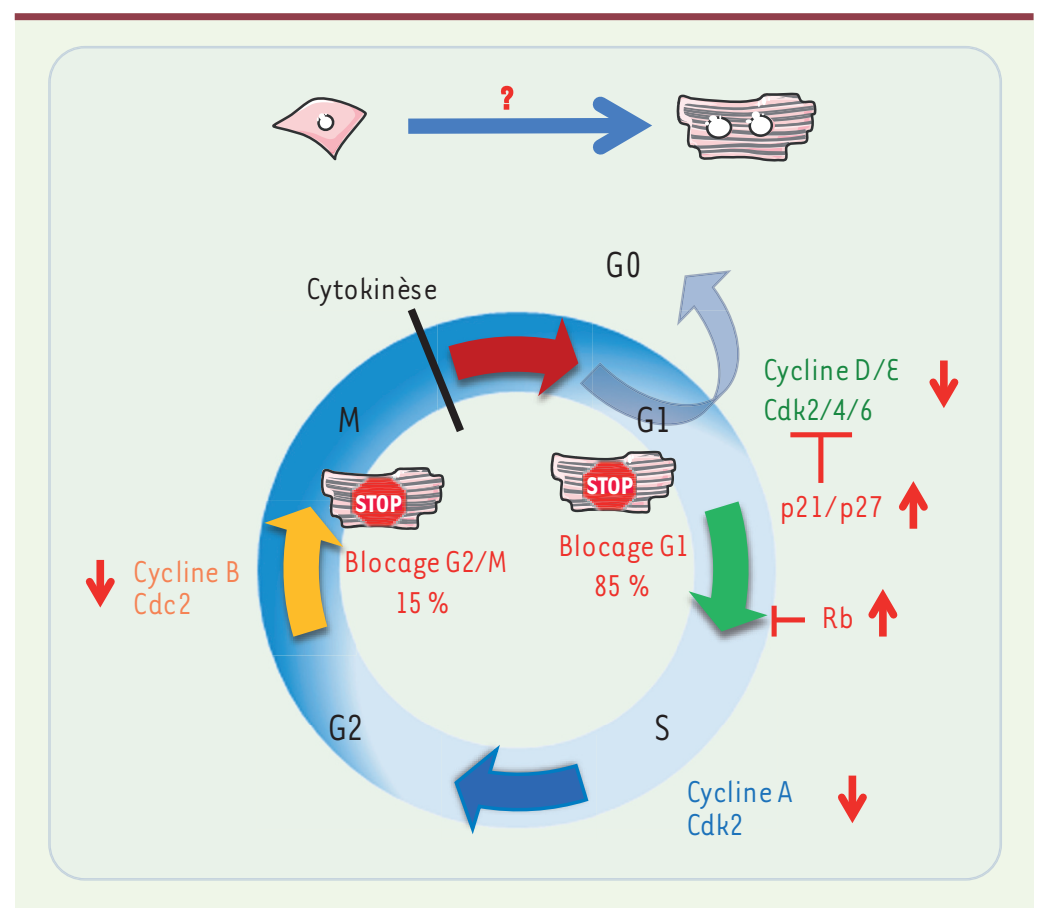

Figure 3. Maturation des cardiomyocytes et cycle cellulaire. Au cours de la maturation cardiaque, les CM présentent plusieurs anomalies du cycle cellulaire (petites flèches rouges), expliquant la production d'une majorité de cellules matures binucléées chez les rongeurs. Ces CM matures binucléés correspondent à un stade de différenciation terminale, postmitotique. Le blocage du cycle se produit en phase G0/G1 (85\%) et également G2/M ( $15 \%$ ) du cycle cellulaire. D'un point de vue mécanistique, au cours de la maturation, les $C M$ répriment les régulateurs positifs du cycle cellulaire (cycline $D / \varepsilon / A / B, C d k 2 / 4 / 6, C d c 2)$, alors qu'ils activent de façon concomitante les inhibiteurs (Rb, p21, p27).

\section{Le réveil des cardiomyocytes}

Si des anomalies du cycle cellulaire au cours de la maturation peuvent expliquer la perte de la capacité de division des CM mammifères adultes, il n'en reste pas moins vrai que les CM matures conservent un potentiel prolifératif, ce dont témoigne leur réponse à la stimulation par des agents mitogènes, NRGl, FGF-l ou périostine [13-15]. Certaines de ces molécules mitogènes sont aussi naturellement sécrétées et sont nécessaires à la cardiogenèse. Elles agissent directement sur les CM (NRGI) ou des structures non musculaires cardiaques, telles que les valves (périostine) [31, 32]. La réponse des CM à ces molécules indique que ces cellules expriment bien les récepteurs spécifiques à ces différents facteurs. Néanmoins, la perte progressive de ces stimulus au cours de la maturation pourrait expliquer l'incapacité des CM matures à proliférer. En accord avec cette hypothèse, une perte de ces facteurs produits au cours du développement du CM a déjà été décrite, en particulier pour des cellules cardiaques non myocytaires avoisinantes [32, 33]. Relancer la production naturelle de ces stimulus pourrait donc représenter une stratégie intéressante et une alternative à l'injection de facteurs mitogènes dont les effets non spécifiques sur d'autres cellules pourraient s'avérer délétères.

\section{Conclusion}

Contrairement au dogme établi jusqu'à présent, il apparaît maintenant clairement que les CM adultes résidents ne sont pas totalement bloqués dans le cycle cellulaire et qu'il est possible de « réveiller » ces cellules. Encore faut-il identifier précisément lesquelles parmi les différentes populations de $C M$ conservent une capacité proliférative et quels processus moléculaires gouvernent cette réactivation. Le taux de renouvellement naturel très faible de ces cellules et la lenteur du processus ne permettent pas actuellement de pallier le dysfonctionnement du tissu cardiaque. Cependant, si l'on devient capables de moduler et d'accélérer pharmacologiquement le renouvellement des CM préexistants dans le tissu cardiaque, peut-être pourra-t-on proposer de nouvelles perspectives thérapeutiques attractives en médecine cardiaque régénératrice, dont le risque serait moindre que celui des stratégies actuelles de thérapie cellulaire. $\diamond$

\section{SUMMARY}

Adult resident cardiomyocytes wake up: new axis for cardiac tissue regeneration

All cardiomyopathies and more specifically myocardial infarction always evolve to cardiomyocytes death and the ensuing heart failure setting. So far, cardiac regenerative medicine has focused on the use of stem cells and completely ignored the resident cardiomyocytes, assumed in a postmitotic state. However, recent findings in zebrafish and mammalians challenge this view and suggest that these cells have some capacity to proliferate and can contribute to heart regeneration. In this review, we propose an overall synthesis about knowledge of the proliferative and regenerative capacities of resident cardiomyocytes, dealing with some mechanistic aspects. In the future, the accurate identification of molecular mechanisms allowing wake-up of resident cardiomyocyte proliferation will certainly open new therapeutic avenues in cardiac regeneration. $\diamond$

\section{REMERCIEMENTS}

Les auteurs remercient vivement le Pr Ducommun (Centre Pierre Potier, Institut des technologies avancées (ITAV), UMS 3039, Toulouse) pour son aide apportée dans la réalisation de ce manuscrit. Notre recherche est financée par la Fondation Lefoulon Delalande, bourse de recherche 2011. 
LIENS D'INTÉRÊT

Les auteurs déclarent n'avoir aucun lien d'intérêt concernant les données publiées dans cet article.

\section{RÉFÉRENCES}

1. Laflamme MA, Murry C $\varepsilon$. Heart regeneration. Nature ; 473 : 326-35.

2. Poss KD, Wilson LG, Keating MT. Heart regeneration in zebrafish. Science $2002 ; 298: 2188-90$.

3. Lepilina A, Coon AN, Kikuchi K, et al. A dynamic epicardial injury response supports progenitor cell activity during zebrafish heart regeneration. Cell $2006 ; 127: 607-19$

4. Ausoni S, Sartore $S$. From fish to amphibians to mammals: in search of novel strategies to optimize cardiac regeneration. J Cell Biol 2009 ; $184: 357-64$

5. Jopling C, Sleep $\varepsilon$, Raya M, et al. Zebrafish heart regeneration occurs by cardiomyocyte dedifferentiation and proliferation. Nature 2010 ; 464 : 606-9.

6. Sleep $\varepsilon$, Boue $S$, Jopling C, et al. Transcriptomics approach to investigate zebrafish heart regeneration. J Cardiovasc Med (Hagerstown) $2010 ; 11: 369-80$.

7. Kikuchi K, Holdway JE, Werdich AA, et al. Primary contribution to zebrafish heart regeneration by gata4(+) cardiomyocytes. Nature $2010 ; 464: 601-5$.

8. Hsieh PC, Segers VF, Davis ME, et al. Evidence from a genetic fate-mapping study that stem cells refresh adult mammalian cardiomyocytes after injury. Nat Med $2007 ; 13: 970-4$.

9. Drenckhahn JD, Schwarz QP, Gray S, et al. Compensatory growth of healthy cardiac cells in the presence of diseased cells restores tissue homeostasis during heart development. Dev Cell 2008 ; 15 : 521-33.

10. Porrello $\varepsilon R$, Mahmoud Al, Simpson $\varepsilon$, et al. Transient regenerative potential of the neonatal mouse heart. Science 2011 ; 331 : 1078-80.

11. Bergmann 0, Bhardwaj RD, Bernard S, et al. Evidence for cardiomyocyte renewal in humans. Science $2009 ; 324$ : 98-102

12. Kajstura J, Urbanek K, Perl S, et al. Cardiomyogenesis in the adult human heart. Circ Res 2010 ; $107: 305-15$.

13. Bersell K, Arab S, Haring B, Kuhn B. Neuregulinl/ErbB4 signaling induces cardiomyocyte proliferation and repair of heart injury. Cell $2009 ; 138: 257-70$.

14. Engel FB, Hsieh PC, Lee RT, Keating MT. FGFI/p38 MAP kinase inhibitor therapy induces cardiomyocyte mitosis, reduces scarring, and rescues function after myocardial infarction. Proc Natl Acad Sci USA 2006 ; 103 : 15546-51.

15. Kuhn B, del Monte F, Hajjar RJ, et al. Periostin induces proliferation of differentiated cardiomyocytes and promotes cardiac repair. Nat Med $2007 ; 13: 962-9$.

16. Gui C, Zhu L, Hu M, et al. Neuregulin- $1 /$ ErbB signaling is impaired in the rat model of diabetic cardiomyopathy. Cardiovasc Pathol $2012 ; 21$ : 414-20.

17. Li F, Wang X, Capasso JM, Gerdes AM. Rapid transition of cardiac myocytes from hyperplasia to hypertrophy during postnatal development. J Mol Cell Cardiol 1996; 28 : 1737-46.
18. Camelliti P, Borg TK, Kohl P. Structural and functional characterisation of cardiac fibroblasts. Cardiovasc Res $2005 ; 65: 40-51$.

19. Piquereau J, Novotova $M$, Fortin D, et al. Postnatal development of mouse heart: formation of energetic microdomains. J Physiol $2010 ; 588: 2443-54$.

20. Peters NS, Severs NJ, Rothery SM, et al. Spatiotemporal relation between gap junctions and fascia adherens junctions during postnatal development of human ventricular myocardium. Circulation 1994 ; 90 : 713-25.

21. Swynghedauw B. Are adult cardiocytes still able to proliferate? Arch Mal Cour Vaiss $2003 ; 96: 1225-30$.

22. Olivetti G, Cigola $\varepsilon$, Maestri R, et al. Aging, cardiac hypertrophy and ischemic cardiomyopathy do not affect the proportion of mononucleated and multinucleated myocytes in the human heart. J Mol Cell Cardiol 1996; 28 : 1463-77.

23. Anversa P, Kajstura J. Ventricular myocytes are not terminally differentiated in the adult mammalian heart. Circ Res $1998 ; 83: 1-14$.

24. Ahuja P, Sdek P, MacLellan WR. Cardiac myocyte cell cycle control in development, disease, and regeneration. Physiol Rev 2007 ; 87 : 521-44.

25. Poolman RA, Brooks $G$. Expressions and activities of cell cycle regulatory molecules during the transition from myocyte hyperplasia to hypertrophy. $J$ Mol Cell Cardiol $1998 ; 30: 2121-35$.

26. Matz DG, Oberpriller J0, Oberpriller JC. Comparison of mitosis in binucleated and mononucleated new cardiac myocytes. Anat Rec $1998 ; 251: 245-55$

27. Poolman RA, Gilchrist R, Brooks G. Cell cycle profiles and expressions of p21CIPl AND P27KIPI during myocyte development. Int J Cardiol $1998 ; 67$ : 133-42.

28. Campa VM, Gutierrez-Lanza R, Cerignoli F, et al. Notch activates cell cycle reentry and progression in quiescent cardiomyocytes. J Cell Biol 2008 ; 183 : 129-41.

29. Porrello $E R$, Johnson $B A$, Aurora $A B$, et al. MiR- 15 family regulates postnatal mitotic arrest of cardiomyocytes. Circ Res $2011 ; 109: 670-9$.

30. Engel FB, Schebesta M, Keating MT. Anillin localization defect in cardiomyocyte binucleation. J Mol Cell Cardiol 2006 ; 41 : 601-12.

31. Norris RA, Moreno-Rodriguez R, Hoffman S, Markwald RR. The many facets of the matricelluar protein periostin during cardiac development, remodeling, and pathophysiology. J Cell Commun Signal 2009; $3: 275-86$.

32. Smith TK, Bader DM. Signals from both sides: control of cardiac development by the endocardium and epicardium. Semin Cell Dev Biol 2007 ; $18: 84-9$

33. leda M, Tsuchihashi T, Ivey KN, et al. Cardiac fibroblasts regulate myocardial proliferation through betal integrin signaling. Dev Cell 2009 ; 16 : 233-44.

\section{TIRÉS À PART}

C. Mias

\section{STRESS, TRAUMATISMES ET INSOMNIES}

\section{de Jean-Pierre FRESCO}

\section{L'insomnie c'est}

1 Français sur 5

1 personne âgée sur 3

qui en souffrent
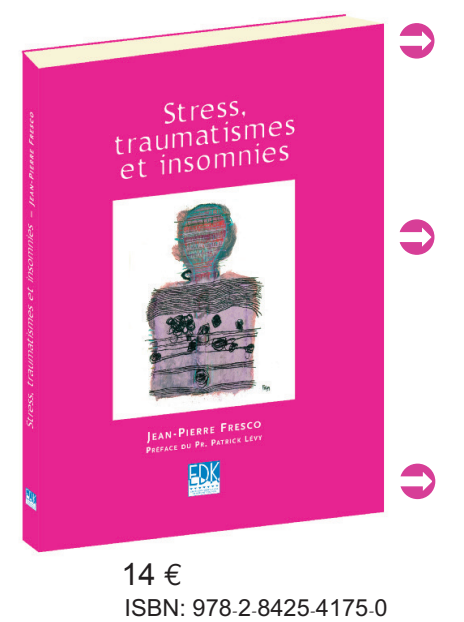

\section{Une étude}

sur les liens entre stress, traumatismes et insomnies.

\section{Une réflexion philosophique et sociétale sur les conditions de travail et de vie d'aujourd'hui.}

\section{Une analyse}

d'un mal fréquent mais encore peu compris.
edpsciences
Ouvrage disponible sur : www.edition-sciences.com 